\title{
Prospective Role of Multicenter Bonding for Efficient and Selective Hydrogen Transport
}

\author{
Daniele Stradi, ${ }^{1,2}$ Francesc Illas, ${ }^{1}$ and Stefan T. Bromley ${ }^{1,3, *}$ \\ ${ }^{1}$ Department de Química Física \& Institut de Química Teórica i Computacional (IQTCUB), Universitat de Barcelona, \\ E-08028 Barcelona, Spain \\ ${ }^{2}$ Dipartimento di Scienze Chimiche, Università degli Studi di Trieste, I-34217, Trieste, Italy \\ ${ }^{3}$ Institució Catalana de Recerca i Estudis Avançats (ICREA), E-08010 Barcelona, Spain \\ (Received 10 December 2009; revised manuscript received 19 May 2010; published 21 July 2010)
}

\begin{abstract}
Multicenter bonding is shown to be able to dramatically reduce atomic transport barriers in solids. Theoretical analysis of $\mathrm{H}$ atoms in a nanoporous polymorph of $\mathrm{ZnO}$ (SOD-ZnO) shows intercage hopping to be aided by four-center bonds which: (i) radically reduce the sterically hindered H-transport barrier to be close to that found in $\mathrm{Pd}$ membranes, and (ii) induce $p$ doping. SOD-ZnO is also shown to be thermodynamically favored under triaxial tension and selective for encapsulating weakly perturbed $\mathrm{H}$ atoms. Such materials have potential use in atomic transport, control, and purification.
\end{abstract}

DOI: 10.1103/PhysRevLett.105.045901

PACS numbers: 66.30.- h, 71.15.Mb, 71.20.Nr

Transport of hydrogen through materials and its interaction with its host while in transit are hugely important with respect to the technological challenges posed by the future use of hydrogen as a clean renewable fuel. Vital for achieving this goal is finding stable materials for purification membranes [1] which can transport hydrogen efficiently and selectively. Currently the most selective membranes are based upon expensive, often mechanically fragile, Pd-based films that transport atomic $\mathrm{H}$ via interstitial sites [2]. The properties of $\mathrm{H}$ atoms in inorganic solids have also been found to lay at the heart of the seemingly unrelated puzzle of understanding the charge doping of $\mathrm{ZnO}$ for harnessing its huge potential in optoelectronics and spintronics [3]. Atomic $\mathrm{H}$ can pass through wurtzite $\mathrm{ZnO}$ (wZ$\mathrm{ZnO})$ via interstitial and midbond $(\mathrm{Zn}-\mathrm{H}-\mathrm{O})$ hopping with a barrier of $\sim 1 \mathrm{eV}[4,5]$, but can be trapped by $\mathrm{O}$ vacancies forming a stable multicenter bond (MCB) [6]. The electronic state of such MCBs provides a plausible explanation for the inherent $n$-type conductivity of wz-ZnO [7]. Herein we investigate $\mathrm{MCB}$ states in a pristine crystalline solid and how they may influence atomic transport. We consider the recently predicted, moderately metastable, SOD-ZnO phase [8] which has a low density porous structure and thus potentially more accommodating of $\mathrm{H}$ atoms than wz-ZnO. Although intended as a proof of principle study, we confirm that SOD-ZnO is thermodynamically favored over likely competing denser phases at modest negative pressures and is hence synthetically viable. Our calculations show that nanocages of SOD-ZnO are selective receptacles for weakly perturbed $\mathrm{H}$ atoms, potentially having application for atomic control [9] and hydrogen purification [1]. We further show that MCBs significantly reduce intercage hopping barriers for atomic $\mathrm{H}$ in SOD-ZnO, while also leading to transient $p$ doping, thus enlarging the potential technological impact of this phenomenon.

All calculations employed periodic density functional theory (DFT) using the VASP code [10]. Valence states were expanded in a plane waves basis with an energy cutoff of
$500 \mathrm{eV}$, with core states described by the projector augmented wave method [11] The cubic SOD-ZnO structure was treated using unit cells (UCs) with a $5 \times 5 \times 5$ Monkhorst-Pack (MP) grid of $k$ points, and with $3 \times 3 \times$ 3 supercells (SCs) containing 324 atoms at the $\Gamma$-point. A single $\mathrm{H}$ atom was initially centered in one SOD-ZnO cage giving a $50 \% \mathrm{H}$ atom per cage filling in the UC case and a $5.5 \%$ filling in the SC calculations. Consistent with previous work [8], we used the PW91 generalized gradient approximation (GGA) functional [12]. Studies have shown improvements to a range of GGA-calculated properties of $\mathrm{wZ}-\mathrm{ZnO}$ with respect to experiment upon including nonlocal Fock exchange [13,14]. Using the HSE [15] rangeseparated hybrid functional we thus incorporated $25 \%$ and $37.5 \%$ of screened Fock exchange (SFE) into the bare PW91 functional (referred to as Fock-25 and Fock-37.5, respectively), using standard HSE screening. Although including 25\% SFE improves the calculated electronic, mechanical, and structural properties of $\mathrm{wz}-\mathrm{ZnO}$ with respect to a pure GGA approach [13], it was shown that increasing to $37.5 \%$ SFE provides an even better match with wz-ZnO experimental data [14]. All computationally costly calculations using SFE used a UC with a $2 \times 2 \times 2$ $\Gamma$-centered MP $k$-point grid. Energy barriers were computed using the climbing image nudged elastic band method (CI-NEB) [16]. Minima and transition states were verified to have zero or only one imaginary vibrational frequency, respectively. Enthalpy versus pressure graphs were derived from Birch-Murnaghan fits to GGAcalculated energy versus volume data using $\Gamma$-point $3 \times$ $3 \times 3$ SC calculations. GGA microcanonical ab initio molecular dynamics (AIMD) calculations were performed for 10 ps using a $2 \times 2 \times 2 \mathrm{SC}$ and a 0.25 fs time step.

$\mathrm{ZnO}$ is experimentally observed almost exclusively in the dense wz-ZnO ground state phase. Recently, it was predicted that a lower density $\mathrm{ZnO}$ phase (BCT-ZnO) could be stabilized in wZ-ZnO nanorods under uniaxial tensions of $\geq 7 \mathrm{GPa}$ [17]. Our GGA calculations confirm 
this result for triaxial tensile stresses (negative pressure) applied to bulk wz-ZnO, but also show that SOD-ZnO is more stable than both $\mathrm{BCT}-\mathrm{ZnO}$ and $\mathrm{wz}-\mathrm{ZnO}$ for stresses $\geq 3.2 \mathrm{GPa}$ (see Fig. 1). To access SOD-ZnO we suggest using $\mathrm{ZnO}$ tetrapods, which have proved to be sources of otherwise difficult to access $\mathrm{ZnO}$ phases [18], and allow for application of tensile stress in four mutually opposing directions.

SOD-ZnO consists of a body-centered cubic array of truncated octahedral $(\mathrm{ZnO})_{12}$ nanocages linked by axially aligned hexagonal $\mathrm{Zn}_{3} \mathrm{O}_{3}$ nanopores (see Fig. 1). The optimized cell parameter gives the internuclear diameter of the cages and is almost identical $(5.655 \pm 0.005 \AA)$ in all calculations, which also consistently confirm SOD-ZnO to be mildly metastable with respect to $\mathrm{wz}-\mathrm{ZnO}$ at zero pressure and $0 \mathrm{~K}(+0.11 \mathrm{eV} / \mathrm{ZnO}$ with Fock-37.5 to $+0.14 \mathrm{eV} / \mathrm{ZnO}$ with GGA). The nanoporous structure of SOD-ZnO suggests potential uses for atomic control and transport. $\mathrm{H}$ atoms placed at the center of the cages reside in energy minima behaving as isolated species having little interaction with SOD-ZnO $(+0.01 \mathrm{eV}$ with PW91 up to $+0.14 \mathrm{eV}$ with Fock-37.5). Displacing $\mathrm{H}$ atoms away from the cage centers to $\leq 40 \%$ of the internuclear distance to the cage wall in all directions (and $\leq 90 \%$, respectively, in nontransport directions - see below) always led to energy increases and a return to the central minima upon release. The H@ZnO cage center minimum was analyzed in a number of ways. Structural deformations induced in the SOD-ZnO structure by $\mathrm{H}$ were negligible $(<0.002 \AA$ w.r.t. empty SOD-ZnO). Bader analysis [19] gave an H-centered charge of $0.95 e$, indicating essentially no charge transfer to or from $\mathrm{H}$ atoms (confirmed by a localized $\mathrm{H}$-centered

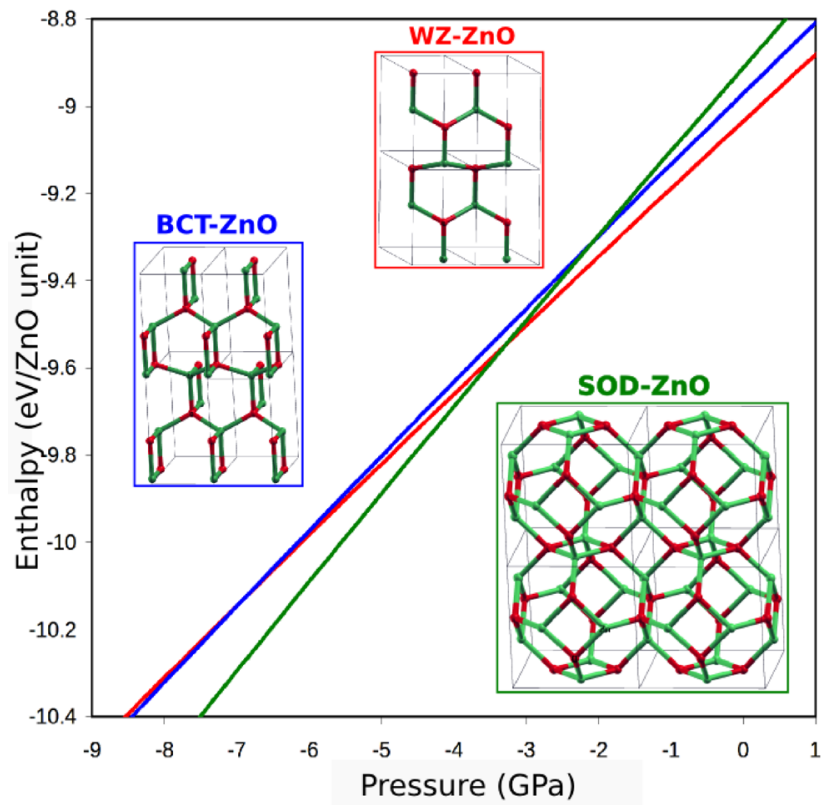

FIG. 1 (color online). Enthalpy vs. pressure curves for wz-ZnO (red), BCT-ZnO (blue) and SOD-ZnO (green). Atom key: Zn green (light gray), $\mathrm{O}$ red (dark gray). spherical charge density). The $\mathrm{H} 1 s$-projected local density of states (LDOS) shows only one significant spin component below the Fermi level, consistent with one electron residing in a $\mathrm{H} 1 s$ orbital. These results (at a GGA level) are summarized in Fig. 2(a). Placing larger atomic species (e.g., He) in the cages resulted in a much stronger repulsive interaction, or ionization and electron transfer to SOD$\mathrm{ZnO}$ (e.g., $\mathrm{Li} \rightarrow \mathrm{Li}^{+}$). At temperatures chosen to limit both thermal hopping and tunneling, H-selective SOD$\mathrm{ZnO}$ nanocages potentially provide an attractive solution for atomic control [9] (e.g., 3D H atom arrays for quantum computing).

We probed the atomic transport properties of SOD-ZnO by following a $\mathrm{H}$ atom along its minimum energy intercage pathway in the [111] crystal direction from a cage center towards, and through, the center of a hexagonal $\mathrm{Zn}_{3} \mathrm{O}_{3}$ nanocage aperture [see Fig. 2]. The diameter of the apertures is $\sim 30 \%$ that of the nanocages and $\mathrm{H}$ transport was expected to be strongly hindered. In a UC/GGA calculation the energetic contribution to the $\mathrm{H}$ transport barrier only due to the distortion of the SOD-ZnO structure yields a maximum energy of $1.34 \mathrm{eV}$ [see Fig. 3(a)]. Remarkably, however, the corresponding total energy of the calculated barrier including the $\mathrm{H}$ atom and its interactions with SOD-ZnO, was found to be only $0.05 \mathrm{eV}$ ( $>96 \%$ reduction). In the $\mathrm{SC}$ case, the barrier further decreases to $0.03 \mathrm{eV}$ [see Fig. 3(b)] due to reduced finite size effects. Specifically, the SOD-ZnO framework can relax more upon $\mathrm{H}$ passage through the ring leading to a lower energy H-SOD-ZnO interaction (a known effect in transport energetics in porous materials [20]). The structural distortions in the $\mathrm{Zn}_{3} \mathrm{O}_{3}$ ring as the $\mathrm{H}$ atom passes through it are shown to be similarly small for all UC calculations in Fig. 3(c) (the reduction in this effect when using a $\mathrm{SC}$ is also shown for the GGA calculation).

The small intercage $\mathrm{H}$ transport barrier in SOD-ZnO implies that a stabilizing interaction offsets the energetically costly $\mathrm{H}$-induced framework distortion. To unravel the origin of this effect, we calculated the $\mathrm{H} 1 s$-projected LDOS at a series of points along the hopping barrier. Approaching the $\mathrm{Zn}_{3} \mathrm{O}_{3}$ ring, two pairs of spin components emerge with well-defined peaks of similar energy, well below and above the Fermi level [see Fig. 2(b)], which can be interpreted as a filled bonding orbital and an empty antibonding orbital. The valence band (VB) top consists of hybridized $\mathrm{Zn}_{3 d^{-}} \mathrm{O}_{2 p}$ states directed along the $\mathrm{Zn}-\mathrm{O}$ bonds. In directions away from $\mathrm{Zn}-\mathrm{O}$ bonds (e.g., the ring center) the potential for orbital overlap is highly diminished and no $\mathrm{H}_{1 s}-\left(\mathrm{Zn}_{3 d^{-}} \mathrm{O}_{2 p}\right)$ interaction appears to take place. Isotropic $s$ states of $\mathrm{Zn}$ and $\mathrm{O}$ are both potentially available but, as the $\mathrm{O}_{2 s}$ states lay very deep energetically, the $\mathrm{H}_{1 s}-\mathrm{Zn}_{4 s}$ interaction is left as most viable. Overlap between the $\mathrm{H}$ $1 s$-projected and $\mathrm{Zn} 4 s$-projected LDOS of the $\mathrm{Zn}$ atoms in the $\mathrm{Zn}_{3} \mathrm{O}_{3}$ ring [see Fig. 2(b)] indeed shows hybridization. In all calculations we also see a MCB Zn-H-shared charge density distribution of the new state [see Fig. 2(b)]. We 

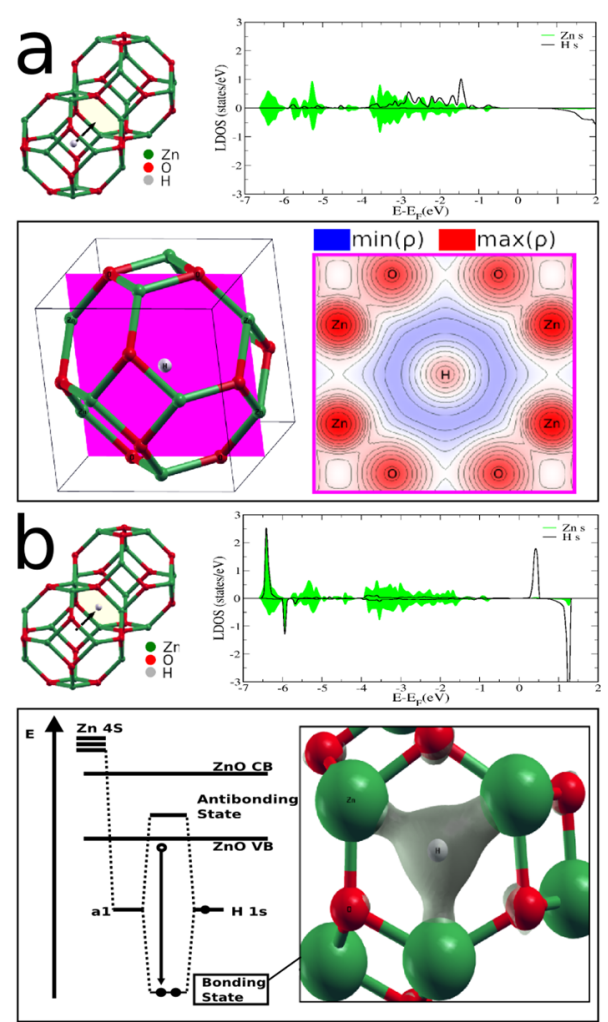

FIG. 2 (color online). (a) Top left: inner cage location of $\mathrm{H}$, top right: spin polarized LDOS, bottom: charge density in highlighted plane, (b) Top left: intercage location of $\mathrm{H}$, top right: spin polarized LDOS, bottom: schematic of electronic levels and charge isodensity $\left(0.03 \mathrm{e}^{-} / \AA^{3}\right)$ of the MCB state. LDOS key: black is $\mathrm{H} 1 s$, green is $\mathrm{Zn} 4 s$.

conclude that a symmetric linear combination of empty $4 \mathrm{~s}$ orbitals of the three $\mathrm{Zn}$ atoms of the $\mathrm{Zn}_{3} \mathrm{O}_{3}$ ring and the $\mathrm{H}_{1 s}$ orbital form a doubly occupied MCB state. One electron in this state comes from the $\mathrm{H}$ atom and the second is transferred from the top of the VB [see Fig. 2(b)]. The $\mathrm{H}_{1 s}-\mathrm{Zn}_{4 s}$ MCB state lies low in energy with respect to the VB maximum, and thus the drop in energy due to the donation of this second electron is the likely origin of the observed stabilizing effect. MCB states of $\mathrm{H}$ atoms in inorganic materials has raised much recent interest [6,21]. For H@wz-ZnO, MCB interactions are also $\mathrm{Zn}_{4 s}-\mathrm{H}_{1 s}$ but are substitutional and give rise to $n$ doping. In $\mathrm{H} @ S O D-Z n O$ the $\mathrm{Zn}_{4 s}-\mathrm{H}_{1 s}$ MCB occurs without vacancy defects and causes the SOD-ZnO framework to be electron deficient, i.e., $p$ doped. Obtaining reliably $p$-doped $\mathrm{ZnO}$ remains an unrealized but much sought after goal of semiconductor research [7]. Here, we highlight a novel link between atomic transport and (transient) $p$ doping in SOD-ZnO, suggesting that diffusing atomic species could play an important role in electronic doping.

In all calculations the SOD-ZnO structure and its energy above $\mathrm{wz}-\mathrm{ZnO}$ is consistently reproduced (see above) in line with the similar performance of pure GGA and HSE calculations of $\mathrm{ZnO}$ phases under pressure [13]. We thus expect the wz-ZnO-to-SOD-ZnO transition pressure (where the relative enthalpy is zero) to be relatively unaffected by SFE. The (relative) energies within a single system (e.g., band gap, barriers), however, can be strongly affected by SFE. Fock- 25 calculations show the $\mathrm{H}$ transport barrier height increases from the pure GGA UCcalculated value of $0.05 \mathrm{eV}$ to $0.36 \mathrm{eV}$, further rising to $0.50 \mathrm{eV}$ when using Fock-37.5 [see Fig. 3(a)] with a concomitant increase of the SOD-ZnO band gap from $1.07 \mathrm{eV}$ (GGA) to $3.65 \mathrm{eV}$ (Fock-37.5). Note that the Fock-37.5 wz$\mathrm{ZnO}$ band gap is $3.50 \mathrm{eV}$, only slightly above experiment $3.44 \mathrm{eV}$ [22], indicating that SFE improves the electronic description. Although the experimental band gap of SOD$\mathrm{ZnO}$ is as yet unknown, it is likely that incorporating between $25 \%$ and $37.5 \%$ SFE provides a better prediction of the electronic structure, and thus an improved estimate of the MCB-stabilized barrier. The MCB-induced stabilization [difference between dashed and solid lines in Fig. 3(a)] also increases with increased SFE (from $1.29 \mathrm{eV}$ with GGA to $1.56 \mathrm{eV}$ with Fock-37.5). These values are comparable to the binding energy per $\mathrm{H}$ atom of dissociatively absorbed $\mathrm{H}_{2}$ in the center of $\mathrm{Zn}_{3}$-based ringlike surface sites of reconstructed $\mathrm{ZnO}$ [0001] oxygenterminated surfaces $(1.01 \mathrm{eV})$ calculated using hybrid DFT methods [23]. This suggests that our predicted MCB stabilization is not specific to SOD- $\mathrm{ZnO}$.

Applying the 44\% UC-to-SC decrease in barrier height, seen when reducing finite size effects in the GGA calculations, we arrive at $\mathrm{H}$ transport barriers of $0.19 \mathrm{eV}$ for Fock-25 and $0.28 \mathrm{eV}$ for Fock-37.5. We note that the GGAcalculated $\mathrm{H}$ diffusion barrier of $\sim 0.5 \mathrm{eV}$ for $\mathrm{wZ}-\mathrm{ZnO}$ [24] may also benefit from inclusion of SFE to improve the agreement with the experimental value of $\sim 1 \mathrm{eV}[4,5]$. The relatively large barrier in $\mathrm{wz}-\mathrm{ZnO}$ is likely due to $\mathrm{H}$ atoms passing through highly constraining cavities, resulting in $\mathrm{Zn}-\mathrm{H}-\mathrm{O}$ bond center positions being most stable. In SOD-ZnO, no stable Zn-H-O minima could be found and all attempts to place $\mathrm{H}$ atoms in midbond locations led to relaxation back to an inner cage minimum, or a rupture of the SOD-ZnO structure forming a $\mathrm{Zn}-\mathrm{OH}$ group. Although framework breaking leads to a GGA energy $\sim 0.85 \mathrm{eV}$ lower than the interior cage minimum, CI-NEB calculations show that this process has a $0 \mathrm{~K}$ energy barrier $\sim 3.5$ times higher than that for intercage hopping through $\mathrm{Zn}_{3} \mathrm{O}_{3}$ rings. Similarly, starting from the top of the hopping barrier, where the SOD-ZnO framework is most distorted, the barrier for $\mathrm{H}$-framework reactions is $\sim 3$ times higher than the intercage hopping barrier. These barrier differences show that intercage $\mathrm{H}$ diffusion is energetically significantly more favored over $\mathrm{Zn}-\mathrm{OH}$ forming reactions. The free energy barriers also include entropic considerations and effects of thermal vibrations which can influence any purely energetic bias. To study finite temperature $\mathrm{H}$ transport in SOD-ZnO we also probed the free energy landscape of the H@SOD-ZnO system by AIMD simulations in the range $150-350 \mathrm{~K}$. In all AIMD runs $\mathrm{H}$ hopping between cages occurred frequently but reactive rupture of SOD$\mathrm{ZnO}$ was never observed. 


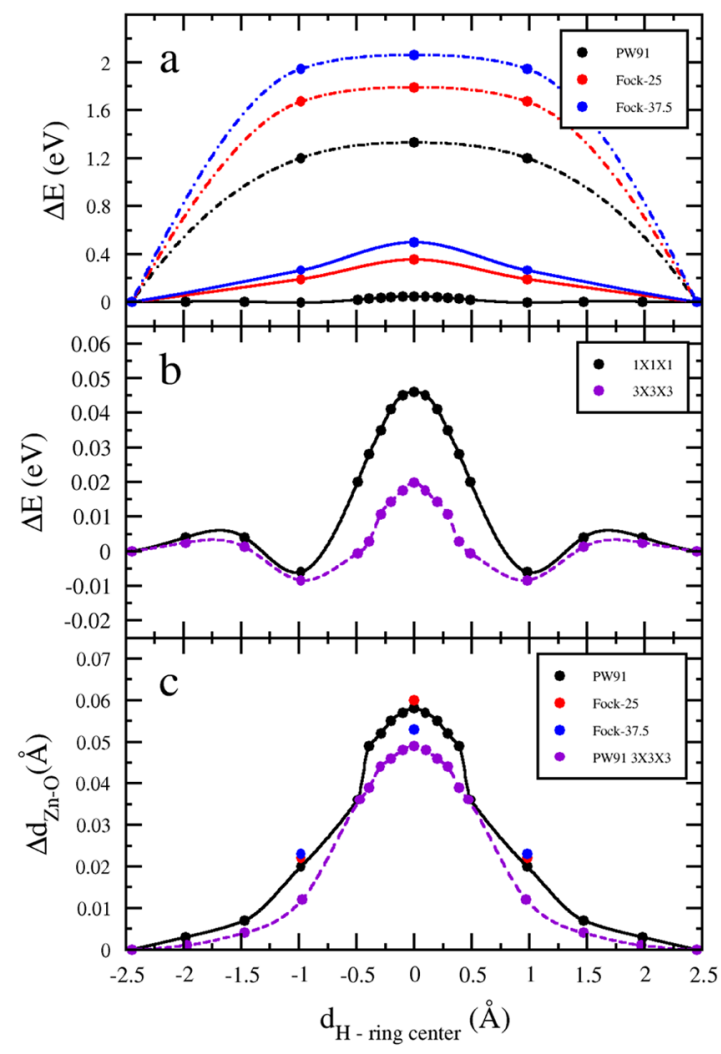

FIG. 3 (color online). (a) Hopping barrier (solid lines), steric effect (dashed lines), (b) barrier reduction: UC (solid line) to SC (dashed line), (c) average $\mathrm{Zn}-\mathrm{O}$ distance changes in the $\mathrm{Zn}_{3} \mathrm{O}_{3}$ ring.

To more practically assess the significance of the smaller $\mathrm{H}$ diffusion barrier in SOD-ZnO with respect to $\mathrm{wz}-\mathrm{ZnO}$, a suitable comparison is the transport barrier of $\mathrm{H}$ through $\mathrm{Pd}$ (widely used in $\mathrm{H}$ purification membranes). GGA functionals generally perform well for metals and the GGAcalculated diffusion barrier of $0.17 \mathrm{eV}$ [25] is probably trustworthy and is comparable with our better estimates of the $\mathrm{H}$ diffusion barrier in SOD-ZnO $(0.19-0.28 \mathrm{eV})$. Although making very selective membranes, $\mathrm{Pd}$ is expensive and mechanically fragile. Our calculations suggest that materials displaying efficient and selective MCBassisted $\mathrm{H}$ transport, such as $\mathrm{SOD}-\mathrm{ZnO}$, could, if suitable preparative methods were found, provide potentially cheap and relatively robust components for $\mathrm{H}$ purification applications. We also note that the highly favorable $\mathrm{H}_{2}$-splitting property of $\mathrm{Pd}$ can also be realized by $\mathrm{ZnO}$ surfaces potentially allowing for $\mathrm{ZnO}$-only membranes which could produce atomic $\mathrm{H}$ from the gas phase.

Using state-of-the-art periodic DFT calculations we show that the nanoporous SOD-ZnO phase is only moderately metastable at zero temperature and pressure, and is likely to be thermodynamically stable under triaxial tensile stress. Because of its atomic and electronic structure, SOD$\mathrm{ZnO}$ is predicted to be able to (i) selectively encapsulate $\mathrm{H}$ atoms at suitably chosen temperatures while only very weakly perturbing their properties, (ii) transport $\mathrm{H}$ atoms with relatively small barriers (and thus at relatively low temperatures with respect to $\mathrm{wz}-\mathrm{ZnO}$ ), due to a MCBassisted hopping mechanism, and (iii) become $p$ doped while transporting $\mathrm{H}$ atoms. This predictive, proof of principle study, highlights a prospective novel role of MCBs which could open the door to a new range of materials for transport, purification, and control of atomic species.

MICINN Grant No. FIS2008-02238, Generalitat de Catalunya Grants No. 2009SGR1041 and XRQTC, and time on Marenostrum via the Barcelona Supercomputing Center are all acknowledged.

*Corresponding author; s.bromley@ub.edu

[1] T. M. Nenoff, R. J. Spontak, and C. M. Aberg, MRS Bull. 31, 735 (2006).

[2] D. S. Scholl and Y.H. Ma, MRS Bull. 31, 770 (2006).

[3] C. Klingshirn, Chem. Phys. Chem. 8, 782 (2007).

[4] N. H. Nickel, Phys. Rev. B 73, 195204 (2006).

[5] K. M. Johansen, J. S. Christensen, E. V. Monakhov, A. Y. Kuznetsov, and B. G. Svensson, Appl. Phys. Lett. 93, 152109 (2008).

[6] A. Janotti and C. G. Van de Walle, Nature Mater. 6, 44 (2007).

[7] M. D. McCluskey and S. J. Jokela, J. Appl. Phys. 106, 071101 (2009).

[8] J. Carrasco, F. Illas, and S. T. Bromley, Phys. Rev. Lett. 99, 235502 (2007).

[9] M. Raizen, Science 324, 1403 (2009).

[10] G. Kresse and J. Hafner, Phys. Rev. B 47, 558 (1993).

[11] P. E. Blöchl, Phys. Rev. B 50, 17953 (1994).

[12] J. P. Perdew and Y. Wang, Phys. Rev. B 45, 13244 (1992).

[13] J. Uddin and G. Scuseria, Phys. Rev. B 74, 245115 (2006).

[14] F. Oba, A. Togo, I. Tanaka, J. Paier, and G. Kresse, Phys. Rev. B 77, 245202 (2008).

[15] J. Heyd, G. E. Scuseria, and M. Ernzerhof, J. Chem. Phys. 118, 8207 (2003); 124, 219906 (2006).

[16] G. Henkelman, B. P. Uberuaga, and H. Jonsson, J. Chem. Phys. 113, 9901 (2000).

[17] A. J. Kulkarni, M. Zhou, K. Sarasamak, and S. Limpijumnong, Phys. Rev. Lett. 97, 105502 (2006).

[18] L. Lazzarini, G. Salviati, F. Fabbri, M. Zha, D. Calestani, A. Zappetini, T. Sekiguchi, and B. Dierre, ACS Nano 3, 3158 (2009).

[19] W. Tang, E. Sanville, and G. Henkelman, J. Phys. Condens. Matter 21, 084204 (2009).

[20] A. W. C. van den Berg, S. T. Bromley, N. Ramsahye, and T. Maschmeyer, J. Phys. Chem. B 108, 5088 (2004).

[21] A. K. Singh, A. Janotti, M. Scheffler, and C. G. Van de Walle, Phys. Rev. Lett. 101, 055502 (2008).

[22] D. C. Reynolds, D. C. Look, B. Jogai, C. W. Litton, G. Cantwell, and W. C. Harsch, Phys. Rev. B 60, 2340 (1999).

[23] S. A. French, A. A. Sokol, S. T. Bromley, C. R. A. Catlow, S. C. Rogers, and P. Sherwood, J. Chem. Phys. 118, 317 (2003).

[24] M. G. Wardle, J. P. Goss, and P. R. Briddon, Phys. Rev. Lett. 96, 205504 (2006).

[25] L. Semidey-Fecha and D. Sholl, J. Chem. Phys. 128, 144701 (2008). 